\title{
Kurzbiographien der Autoren
}

Amend-Traut, Anja, Professorin für Dt. u. Europ. Rechtsgeschichte, Kirchenrecht u. Bürgerliches Recht, Julius-Maximilians-Universität Würzburg. Zuletzt erschienen u. a.: Wechselverbindlichkeiten vor dem RKG. Praktiziertes Zivilrecht in der Frühen Neuzeit, 2009; Der Reichshofrat u. die Kapitalgesellschaften. Die Bemühungen um eine Handelscompanie zwischen den Hansestädten u. Spanien, in: A. Amend-Traut/A. Cordes/W. Sellert (Hrsg.), Geld u. Gerechtigkeit, 2012 (im Ersch.); Zur Geschichte des Notariats in Frankfurt a.M. Das Notariat zwischen Reichskonformität u. kaufmännischen Sonderinteressen, in: Hdb. zur Geschichte des dt. Notariats seit der Reichsnotariatsordnung von 1512, 2012, 315-349.

BORDe, CHRISTIAN, Maître de Conférences en Histoire contemporaine à l'Université du Littoral Côte d'Opale (ULCO: Dunkerque, Calais, Saint-Omer, Boulogne-sur-Mer). Thèse de doctorat d'Université: Le port de Calais, 1814-1914, Université Charles de Gaulle Lille III, 1995. Il publie en 2009: C. Borde, «Le contrebandier, le tulliste et le négociant: Calais, relais européen de l'industrie dentellière, 1802-1832», dans: S. Curveiller, L. Buchard (éds.), Se déplacer du Moyen âge à nos jours, Actes du 6e colloque européen de Calais 2006-2007, Calais, 2009, 291-302.

CORDES, Albrecht, Professor für mittelalterliche u. neuere Rechtsgeschichte u. für Zivilrecht an der Goethe-Universität Frankfurt a.M. Hauptantragsteller des LOEWE-Schwerpunkts „Außergerichtliche u. gerichtliche Konfliktlösung“; Vorstandsmitglied im Hansischen Geschichtsverein; Leiter des Wissenschaftlichen Beirats der Gesellschaft für Reichskammergerichtsforschung; Mitherausgeber der 2. Aufl. des Handwörterbuchs zur deutschen Rechtsgeschichte (HRG). Zuletzt erschienen: Art. Kaufmann, Kaufleute, in: HRG II, 2. Aufl. 2012, 16831690; Acceptance and Rejection of „Foreign“ Legal Doctrine by the Council of Lubeck around 1500, in: S. Dauchy u.a. (Hrsg.) Ratio decidendi, Bd. 2, Berlin 2010, 17-35.

Garnier, Florent, Professeur d'Histoire du droit à l'Ecole de Droit - Université d'Auvergne. Il est l'auteur notamment des articles «Eugène-Edmond Thaller et les Annales de droit commercial»; Le renouveau de la doctrine française. Les grands auteurs de la pensée juridique au tournant du $\mathrm{XX}^{\mathrm{e}}$ siècle. Études réunies par $\mathrm{N}$. Hakim et F. Melleray, Dalloz, coll. Méthodes du droit, Paris 2009, 159-196; «De la coutume et des usages dans la doctrine commerciale française à la fin XIXe siècle et au début XXe siècle», Quaderni Fiorentini, XLI (2012), 299-327. 
Hamon, Thierry, Maître de Conférences en Histoire du Droit à l'Université Rennes 1, Directeur de l'Antenne délocalisée de la Faculté de Droit de Rennes à Saint-Brieuc. Publications significatives: Contributions au Dictionnaire Historique des Juristes Français (Dir: Arabeyre, Halpérin, Krynen), Paris, 2007; «Le statut juridique des routoirs en Bretagne, de l'Ancien Régime au XIXème siècle», dans: Du lin à la toile, Rennes, 2008, 65-91; «L'adjuration à Saint Yves de Vérité, persistance tardive d'une ordalie populaire bretonne», Mémoires de la S.H.A.B. Rennes, 2008, T. 86, 41-88; «L'exploitation des terres agricoles en Droit coutumier breton (XIVème-XIX ̀̀me siècle)», dans: Terre, Forêt et Droit, Nancy, 2006, 273 318.

Hilaire, Jean, Professeur d'histoire du droit, doyen honoraire de la Faculté de droit de l'université Descartes (Paris V), professeur émérite de l'Université Panthéon-Assas (Paris II) depuis 1994. Spécialisations: droit de la famille, procédure civile, droit commercial.

MarZagalli, Silvia, Full-time Professor of Early Modern History at the University of Nice, France. Principal investigator of the program Navigocorpus, ANR grant 2007-2011 (http://navigocorpus.org). Author of "Les boulevards de la fraude». Le négoce maritime et le Blocus continental, 1806-1813. Bordeaux, Hambourg, Livourne, (Villeneuve d'Ascq, 1999); Révolution Consulat et Empire with M. Biard and P. Bourdin (Paris, 2009); Atlas de la Révolution française. Circulations des hommes et des idées, 1770-1804, with P.-Y. Beaurepaire (Paris, 2010); «Rough Waters. The United States involvement in the Mediterranean, $18^{\text {th-19t }} \mathrm{c} . »$, with J. McCusker and J. Sofka (St. John's, Newfoundland, 2010).

MÜsSIG, UlRIKE, Professorin an der Juristischen Fakultät der Universität Passau, Lehrstuhl für Bürgerliches Recht sowie Dt. u. Europ. Rechtsgeschichte. Zuletzt erschienen: Die europ. Verfassungsdiskussion des 18. Jh., Tübingen 2008; Recht u. Justizhoheit, Der gesetzliche Richter im historischen Vergleich von der Kanonistik bis zur Europ. Menschenrechtskonvention, Berlin 22009; La concentration monarchique du pouvoir et la diffusion des modèles constitutionnels français en Europe après 1800, Revue historique de droit français et étranger 88 (2010), 295310; L'ouverture du mouvement constitutionnel après 1830: à la recherche d'un équilibre entre la souveraineté monarchique et la souveraineté populaire, Tijdschrift voor Rechtsgeschiedenis 79 (2011), 489-519; L'avant-garde allemande du discours constitutionnel en $18^{\mathrm{e}}$ sciècle: Entre la Raison d'État et le droit de codécision rationelle, Revue historique de droit français et étranger 90 (2012), 67-84.

Oestmann, Peter, Professor für Bürgerliches Recht u. Dt. Rechtsgeschichte in Münster; Hauptantragsteller des Exzellenzclusters Religion u. Politik; Mitglied der Nordrhein-Westfälischen Akademie der Wissenschaften u. Künste. Zuletzt erschienen: Geistliche u. weltliche Gerichte im Alten Reich (Köln u.a. 2012); Pro- 
zesse aus Hansestädten vor dem Königs- u. Hofgericht in der Zeit bis 1400, in: Zeitschrift der Savigny-Stiftung für Rechtsgeschichte, GA 128 (2011), 114-168.

PAHLOw, LouIS, Professor für Neuere Rechtsgeschichte, Juristische Zeitgeschichte u. Zivilrecht an der Goethe-Universität Frankfurt a.M. Veröffentlichungen zur Geschichte des Privat- u. Wirtschaftsrechts im 19. u. 20. Jh., u. a.: Die zeitliche Dimension des Rechts. Historische Rechtsforschung u. geschichtliche Rechtswissenschaft (2005); Aktienrecht u. Aktiengesellschaft zwischen Revolution u. Reichsgründung, in: Bayer/Habersack (Hrsg.), Aktienrecht im Wandel (2007); Das aus der Ruhe gerissene Recht. Wirtschaftsrecht zwischen Rechtswissenschaft u. Politik, in: Reulecke/Roelcke (Hrsg.), Wissenschaften im 20. Jh. (2008); Grundlagen u. Grundfragen des Geistigen Eigentums (2008); Der Staat als Wächter über die Kreditwirtschaft, in: Zeitschrift für Neuere Rechtsgeschichte 34 (2012).

Pfister-Langanay, Christian, Maître de conférences en histoire moderne à l'Université du Littoral-Côte d'Opale. Sa thèse, sortie en 1985, porte sur le commerce du port de Dunkerque entre 1662 et 1792. Il publie en 2005 une histoire de la construction navale dunkerquoise du XVIIe siècle à 1914. Travaille actuellement sur l'Amirauté de France dans le cadre du programme Navigocorpus, ANR depuis 2007.

Pourchasse, PierricK, Senior Lecturer and Research Fellow in the Department of History at the University of Bretagne Occidentale in Brest. One of the coordinators of Navigocorpus, ANR (a project which aims to compile data on merchant shipping between 1650 and 1850 and put it at public disposal). Representative publication: Le commerce du Nord. Les échanges commerciaux entre la France et l'Europe septentrionale au XVIIIe siècle (Rennes: PUR, 2006).

Rothweiler née RéHAult, AnNe-Estelle, Maître de conférences à l'Université de Strasbourg, 'Le naufrage - les conséquences juridiques de l'infortune de mer en France et en Angleterre du XVIè au XIX è siècle، (Atelier national de reproduction des thèses, 2002); Von der Compagnie de commerce zur société anonyme: Die Geschichte der Aktienrechtsgesellschaft in Frankreich bis zum Code de commerce, in: Bayer/Habersack (Hrsg.), Aktienrecht im Wandel (2007) Bd. 1, 23-45; 'L'assurance des captifs chrétiens au XVIIè siècle', dans: Navires et gens de mer du Moyen Age à nos jours, P. Sturmel (Dir.), (L'Harmattan, 2011), 75-95.

Sachs, Stephen E., Assistant Professor of Law, Duke University School of Law. Representative publications: Constitutional Backdrops, 80 G.W. L. Rev. (forthcoming 2012); Full Faith and Credit in the Early Congress, 95 Va. L. Rev. 1201 (2009); From St. Ives to Cyberspace: The Modern Distortion of the Medieval 'Law Merchant', 21 American University International Law Review 685 (2006). 
Scherner, Karl Otto, bis 1999 Inhaber des Lehrstuhls für Dt. u. Europ. Rechtsgeschichte u. Bürgerliches Recht an der Universität Mannheim. Zuletzt erschienen: Handelsrecht. Selbstregulierung u. Regulierung beim Eisenbahnfrachtgeschäft $u$. im Versicherungswesen in der erste Hälfte des 19. Jh., in: Selbstregulierung im 19. Jh. zwischen Autonomie u. staatlichen Steuerungsansprüchen (Hrsg. v. P. Collin, M. Stolleis u. a.), Frankfurt 2011, 195-229; Artt. Handelsrecht, Kauf, Kommissionsgeschäft, in: HRG 2. Aufl., Bd. 2 (2012) 714-732, 1665-1679, 19881991.

Wijffels, Alain, Professor für Rechtsgeschichte u. Rechtsvergleichung an den Universitäten zu Leiden (NL) u. Louvain-la-Neuve (B), Forschungsdirektor am CNRS (Frankreich). Forschungsthemen: Alte Gerichtsbarkeit, Ius Commune, Kodifikation, Verfassungsgeschichte, Völkerrechtsgeschichte. Kurzlehrbuch: Introduction Historique au Droit. France, Allemagne, Angleterre (Paris, Coll. Thémis Droit, 2010). 


\section{Schriften des Historischen Kollegs:}

Eine Übersicht über die Bände findet sich unter:

www.oldenbourg-wissenschaftsverlag.de/shk

Alle Publikationen des Historischen Kollegs sind verzeichnet unter:

www.historischeskolleg.de

61 Helmut Altrichter (Hrsg.): GegenErinnerung. Geschichte als politisches Argument im Transformationsprozeß Ost-, Ostmittel- und Südosteuropas, 2006, XXII, 326 S. ISBN 3-486-57873-1

62 Jürgen Trabant (Hrsg.): Sprache der Geschichte, 2005, XXIV, 166 S. ISBN 3-486-57572-4

63 Anselm Doering-Manteuffel (Hrsg.): Strukturmerkmale der deutschen Geschichte des 20. Jahrhunderts, 2006, VIII, 273 S. ISBN 3-486-58057-4

64 Jan-Dirk Müller (Hrsg.): Text und Kontext: Fallstudien und theoretische Begründungen einer kulturwissenschaftlich angeleiteten Mediävistik, 2007, XIII, 272 S. ISBN 3-486-58106-5

65 Peter Schäfer (Hrsg.): Wege mystischer Gotteserfahrung. Judentum, Christentum und Islam/Mystical Approaches to God. Judaism, Christianity, and Islam, 2006, $\mathrm{X}, 164$ S. ISBN 3-486-58006-X

66 Friedrich Wilhelm Graf(Hrsg.): Intellektuellen-Götter. Das religiöse Laboratorium der klassischen Moderne, 2009, XIII, 161 S. ISBN 978-3-486-58257-4

67 Werner Busch (Hrsg.): Verfeinertes Sehen. Optik und Farbe im 18. und frühen 19. Jahrhundert, 2008, X, 228 S. ISBN 978-3-486-58490-5

68 Kaspar von Greyerz (Hrsg.): Selbstzeugnissse in der Frühen Neuzeit. Individualisierungsweisen in interdisziplinärer Perspektive, 2007, VII, 201 S. ISBN 978-3-486-58236-9

69 Wilfried Hartmann (Hrsg.): Recht und Gericht in Kirche und Welt um 900, 2007, IX, 249 S. ISBN 978-3-486-58147-8

70 Heinz Schilling (Hrsg.): Konfessioneller Fundamentalismus. Religion als politischer Faktor im europäischen Mächtesystem um 1600, 2007, XI, 320 S. ISBN 978-3-486-58150-8

71 Michael Toch (Hrsg.): Wirtschaftsgeschichte der mittelalterlichen Juden. Fragen und Einschätzungen, 2008, X, 218 S. ISBN 978-3-486-58670-1

72 Tilman Nagel (Hrsg.): Der Koran und sein religiöses und kulturelles Umfeld, 2010, XXIV, 250 S. ISBN 978-3-486-59052-4

73 Karl-Joachim Hölkeskamp (Hrsg.): Eine politische Kultur (in) der Krise? Die „letzte Generation“ der römischen Republik, 2009, XII, 222 S. ISBN 978-3-486-59053-1

74 Karl Schlögel (Hrsg.): Mastering Space. Raum und Raumbewältigung als Probleme der russischen Geschichte, 2011, VIII, 314 S. ISBN 978-3-486-70445-7 


\section{Schriften des Historischen Kollegs:}

75 Aloys Winterling (Hrsg.): Zwischen Strukturgeschichte und Biographie. Probleme und Perspektiven einer römischen Kaisergeschichte (Augustus bis Commodus), 2011, X, 350 S. ISBN 978-3-486-70454-9

76 Rüdiger vom Bruch (Hrsg.): Die Berliner Universität im Kontext der deutschen Universitätslandschaft nach 1800, um 1860 und um 1910, 2010, XVIII, 259 S. ISBN 978-3-486-59710-3

77 Christoph Buchheim und Marcel Boldorf(Hrsg.): Europäische Volkswirtschaften unter deutscher Hegemonie 1938-1945, 2012. X, 270 S. ISBN 978-3-486-70950-6

78 Klaus Schreiner (Hrsg.): Heilige Kriege. Religiöse Begründungen militärischer Gewaltanwendung: Judentum, Christentum und Islam im Vergleich. Kolloquium der Mitglieder des Historischen Kollegs, 7.-9. November 2007. 2008, XIII, 223 S. ISBN 978-3-486-58490-5

79 Jörg Fisch (Hrsg.): Die Verteilung der Welt. Selbstbestimmung und das Selbstbestimmungsrecht der Völker. The World Divided. Self-Determination and the Right of Peoples to Self-Determination, 2011, XXII, 344 S.

ISBN 978-3-486-70384-9

80 Georg Schmidt (Hrsg.): Die deutsche Nation im frühneuzeitlichen Europa. Politische Ordnung und kulturelle Identität? 2010, XIV, 344 S. ISBN 978-3-486-59740-0

81 Albrecht Cordes, Serge Dauchy (Hrsg.): Eine Grenze in Bewegung. Öffentliche und private Justiz im Handels- und Seerecht, 2013, XII, 366 S. ISBN 978-3-486-71799-0

82 Lothar Gall, Dietmar Willoweit (Hrsg.): Judaism, Christianity, and Islam in the Course of History: Exchange and Conflicts, 2011, XII, 470 S. ISBN 978-3-486-59707-3

83 Thomas Welskopp, Alan Lessoff (Hrsg.): Fractured Modernity. America Confronts Modern Times, 1890s to 1940s, 2012, X, 242 S. ISBN 978-3-486-71695-5

84 Gian Luca Potestà (Hrsg.): Autorität und Wahrheit. Kirchliche Vorstellungen, Normen und Verfahren (XIII.-XV. Jahrhundert), 2012, XII, $200 \mathrm{~S}$.

ISBN 978-3-486-70771-1

85 Egon Flaig (Hrsg.): Genesis und Dynamiken der Mehrheitsentscheidung (in Vorbereitung)

86 Heribert Müller (Hrsg.): Das Ende des konziliaren Zeitalters (1440-1450): Versuch einer Bilanz, 2012, X, 352 S. ISBN 978-3-486-71421-0

87 Gangolf Hübinger (Hrsg.): Europäische Wissenschaftskulturen und politische Ordnungen in der Moderne (1890-1970) (in Vorbereitung)

88 Werner Plumpe (Hrsg.): Unternehmer - Fakten und Fiktionen (in Vorbereitung)

\section{Oldenbourg}





